\title{
Fazit und Ausblick
}

Das vorliegende Buch setzt sich mit der neuen Thematik der evolutorischen Finanzökonomie auseinander. Aufbauend auf einer historischen Analyse bisheriger theoretischer Finanzansätze stellt es einen Zusammenhang zwischen biologischen bzw. darwinistischen Konzepten und der Finanzökonomie her. Neue Fragestellungen, bspw. ob Variabilität und Mutation gut für den Finanzmarkt sind oder die Effizienzmarkthypothese durch die Symbiose von Momentumund Value-Strategien einerseits sowie den Begriff der Entropie in Systemen andererseits in einem neuen Licht gesehen werden kann, können mithilfe der evolutorischen Finanzökonomie auf einen neuen Kenntnisstand gehoben werden.

Ebenso wird gezeigt, dass verschiedene institutionelle Teilnehmer des Finanzmarktes und deren Verhaltensweisen zielführend durch evolutorische Annahmen bzw. Denkansätze analysiert werden können. Für Investoren, Unternehmen oder die Aufsicht ergeben sich dadurch neue Handlungsmöglichkeiten, um sich im System des Finanzmarktes erkenntnisleitender zu bewegen.

Es bleibt zu hoffen, dass durch die Verknüpfung von Biologie und Ökonomie die Zukunft und das Verständnis der Finanzökonomie weiter verbessert wird. Dazu kann auch die Integration der Erkenntnisse aus dem Buch in die akademische Lehre beitragen. 